\title{
Technology and evidence-based care enhance postoperative management of chest drains
}

\author{
Daniel G. French ${ }^{1}$, Sebastien Gilbert ${ }^{2}$ \\ ${ }^{1}$ Division of Thoracic Surgery, Dalhousie University, Queen Elizabeth II Hospital-Victoria Campus, Halifax, NS, Canada; ${ }^{2}$ Division of Thoracic \\ Surgery, University of Ottawa, The Ottawa Hospital-General Campus, Ottawa, ON, Canada \\ Correspondence to: Sebastien Gilbert, MD. Division of Thoracic Surgery, The Ottawa Hospital, General Campus Suite 6363, 501 Smyth Road, \\ Ottawa, ON K1H 8L6, Canada. Email: sgilbert@toh.on.ca. \\ Comment on: Gao S, Zhang Z, Aragón J, et al. The Society for Translational Medicine: clinical practice guidelines for the postoperative management \\ of chest tube for patients undergoing lobectomy. J Thorac Dis 2017;9:3255-64.
}

Submitted Nov 18, 2018. Accepted for publication Nov 21, 2018.

doi: $10.21037 /$ jtd.2018.11.99

View this article at: http://dx.doi.org/10.21037/jtd.2018.11.99

Despite being considered a routine task by many thoracic surgeons, managing a patient's chest following pulmonary resection remains an evolving science. Enhanced recover after surgery (ERAS) programs strive to standardize surgical care with recent clinical data, advances in technology, and protocolized postoperative care. In addition, to promote consistency within the literature in the setting of advancements in technology, the European Society of Thoracic Surgeons (ESTS), the American Association for Thoracic Surgery (AATS), the Society of Thoracic Surgeons (STS), and the General Thoracic Surgery Club (GTSC) have standardized the terminology related to chest tube management decisions. The term "applied external suction" is defined as application of negative pressure to the pleural space and "no applied external suction" is defined as the absence of applied negative pressure to the pleural space (1).

The management of chest tubes after pulmonary resection begins in the operating room when the number of chest drains left in the thorax is determined. Traditionally, two drains were left: an apical chest tube to drain air and a basilar chest tube to drain liquid. However, when challenged in the literature, four randomized controlled trials (RCTs) (2-5), one non-RCT $(6)$, and two meta-analyses $(7,8)$ found no benefit to leaving a second chest tube in place. As a result, most ERAS protocols leave only one chest tube following a pulmonary resection (9).

Once the patient leaves the operating room chest tube management continues in the recovery room and ward.
With the development of minimally invasive techniques through video assisted thoracoscopic surgery (VATS) and enhanced recovered through ERAS programs, an indwelling chest tube is often the factor limiting discharge from hospital. A single chest tube is left to drain both liquid and air. Liquid is typically lymphatic in nature due to the disruption of the lymphatics and inflammation associated with the pulmonary dissection. Air is drained from an alveolar-pleural fistula.

Traditionally, chest X-rays (CXR) were routinely ordered in the postoperative period. A recent meta-analysis of critical care literature (10) and a retrospective review of prospectively collected data (11) concluded that findings on a routine CXR rarely change patient management in the absence of associated clinical findings. Furthermore, ERAS and standardized chest tube management pathways suggest that tolerating a pneumothorax in an asymptomatic patient is safe $(10,12)$. Despite the lack of high quality evidence in the form of RCTs, this retrospective evidence suggests that the use of on-demand CXR be implemented. In this management approach, a CXR is not routinely ordered in the postoperative period but only in the setting of clinical findings, promoting efficient patient care and decreasing discomfort associated with patient positioning for a CXR.

The maximum liquid output in a 24 -hour period considered safe to discontinue pleural drainage is debated (11). Over the past 12 years, the volume of liquid drainage in a 24-hour period considered safe to remove a chest drain has gradually increased based on the results of trials comparing 
different thresholds. Published trials report on the duration of chest drainage, length of stay (LOS), and the need for thoracentesis or re-insertion of drains. The first RCT in 2002 reported safe removal of chest drains with $200 \mathrm{~mL} / 24$ hours (13). This study was followed by several retrospective reviews looking at much higher thresholds of $400-450 \mathrm{~mL} / 24$ hours $(14,15)$. A more recent prospective study favored using a threshold of $500 \mathrm{~mL} / 24$ hours (16). However, these higher volume thresholds have not been not supported in other RCTs, which suggested that chest tube removal should be delayed until liquid output is less than or equal to $300 \mathrm{~mL} / 24$ hours $(17,18)$. The safe volume of liquid to remove a chest drain is likely between $300-400 \mathrm{cc} / 24$ hours depending on the lean body mass of the patient (11).

Determining the presence or absence of a clinically significant alveolar-pleural fistula is fundamental to making safe decisions surrounding the management of a chest tube. Initial chest drainage devices were based on a threebottle system. This evolved into the currently available analogue drainage systems which combine the collection, water seal, and suction chambers into a single unit. The presence or absence of an air leak is determined by observing bubbling in the water seal chamber. However, this method to assess an air leak is subjective and limited by high inter-observer variability (19). Newer digital devices are commercially available such as the Thopaz (Medela Healthcare, Baar, Switzerland) and Atmos (MedizinTechnik, Germany) drainage systems. These devices provide suction independent of an external source for negative pressure and continuously measure airflow to provide a plot of airflow versus time. Ten RCTs comparing digital and analog chest drainage devices have been conducted (20-29). Six trials concluded that the digital devices resulted in shorter chest tube duration and shorter LOS (20-25). One study showed shorter chest tube duration with no significant difference in LOS (25). Three trials did not find a significant differences in either chest tube duration or LOS (27-29). Two RCTs determined that digital devices resulted in fewer clamping trials $(28,29)$. Variability in chest tube management protocols and delays in removal of chest drains likely explain the inconsistent findings in these studies. It is hopeful that objective measurement of airflow will allow the nursing staff to be integrated into well designed protocols and promote early removal of chest tubes leading to earlier discharge from hospital.

Despite best prevention efforts, prolonged air leaks (PAL) will occur and remain the most common complication after pulmonary resection (30). A PAL is defined as an air leak lasting greater than 5 days. Methods to shorten the duration of an air leak include the use of applied external suction and invasive maneuvers including chemical pleurodesis, bronchial valves, and re-operation. With the development of ERAS programs, acceptance of a PAL with early discharge with a portal device is becoming more common. In general the more recent literature has focused on conservative management of air leaks including tolerating pneumothorax on the CXR and early discharge with a PAL (12). However, invasive maneuvers may be required for patients unable to tolerate an air leak. Furthermore, new and old techniques should be continually explored to find methods to reduce the duration of air leaks and chest tube drainage.

The role of applied external suction has long been debated in the thoracic literature. Two physiologic mechanisms have been proposed to debate the role of applied external suction to help an air leak heal. The first suggests that applied external suction promotes airflow through the fistula, preventing it from healing. This proposed mechanism suggests that an air leak will heal faster without applied external suction. A contradictory mechanism suggests that suction promotes apposition between the parietal and visceral pleural and thus promotes sealing of the air leak. Unfortunately, clinical data in the form of seven RCTs and three meta-analyses have not determined the optimal use of applied external suction. Trials typically compare the incidence of PAL, duration of chest tube, LOS, and presence of a residual pneumothorax with and without applied external suction. Two RCTs and one meta-analysis favored no applied external suction (31-33), while three RCTs and two meta-analyses showed no difference in these outcomes with or without applied external suction (34-38). Explanations for the inconsistent findings have been previously proposed (11): (I) if the true benefit with or without applied external suction is small, inconsistencies between studies may not detect a true difference; (II) assessments of air leaks has been shown to be inconsistent even with experienced clinical teams (19); (III) lastly, there may be no physiologic benefit or risk to applied external suction after pulmonary resection. Acknowledging the controversies, two programs aimed at standardizing air leak management after pulmonary resection have set a regimen of setting chest drains to applied external suction until postoperative day (POD) 1 , then moving to no applied external suction unless there is an adverse event $(9,12)$. With consistent protocolized care offered by ERAS programs and more objective assessment 
of air leaks offered by digital drains, large multi-center RCT may help establish the best regimen for the use of applied external suction in the postoperative period. For now, a consistent approach at an institutional level is likely more important than prolonged debates regarding the benefits and harms of applied external suction.

If the visceral and parietal pleura are opposed in the setting of an air leak, pleurodesis can be achieved with a chemical substance (talc, silver nitrate, doxycycline, tetracycline bleomycin, and interferon) or autologous blood may be administered through an indwelling chest tube at the bedside to induce inflammation and sclerosis leading to adhesions. The literature supporting the use of chemical pleurodesis for postoperative PAL is limited $(39,40)$, however two systematic reviews suggest autologous blood has the potential to reduce the duration of an air leak but more research is needed $(39,41,42)$. The use of bronchial valves to treat postoperative air leaks is presently limited. Although not specific to postoperative air leaks, a multicenter prospective RCT called "valves against standard therapy (VAST)", which will compare bronchial valves to standard management of air leaks, is currently enrolling patients $(43,44)$. For the time being, bronchial valves should remain a last resort for management of postoperative air leaks. Despite the description of multiple intraoperative maneuvers to prevent air leaks such as buttressing the staple line, topical sealants, pleural tent, and induction of pneumoperitoneum, there are no trials comparing reoperation to other methods of managing postoperative air leaks (45). ERAS protocols for pulmonary resection have not included re-operation but tend to focus on more conservative management approaches to treating of air leaks (9). Similar to bronchial valves, re-operation should be limited to few patients and selectively used in the very early postoperative period.

There is sufficient literature to show air leaks can be safely managed in the postoperative period with portable devices (46-48). The most commonly used portable devices are a Heimlich valve and a Pneumostat (Atrium, Hudson, USA) (49). Heimlich valves allow air to escape but prevent re-entry using a rubber one-way valve. A Pneumostat has a one-way valve and a collection chamber to capture effluent. Patients are typically discharged between POD 4 and 5 with a portable device to manage a PAL with follow-up within 3 to 7 days in an outpatient clinic.

Chest tube manage is evolving into a more precise science with increasing evidence to guide chest tube management. New technologies to measure and possible stop air leaks are being explored. Future efforts will include promoting the early identification of patients expected to require discharge with a portable device and prediction models to estimate the day that the air leak will resolve. This will facilitate earlier discharge with a portable device and scheduling of follow-up with the goal of reducing health care costs.

\section{Acknowledgements}

None.

\section{Footnote}

Conflicts of Interest: The authors have no conflicts of interest to declare.

\section{References}

1. Brunelli A, Beretta E, Cassivi SD, et al. Consensus definitions to promote an evidence-based approach to management of the pleural space. A collaborative proposal by ESTS, AATS, STS, and GTSC. Eur J Cardiothorac Surg 2011;40:291-7.

2. Gómez-Caro A, Roca MJ, Torres J, et al. Successful use of a single chest drain post lobectomy instead of two classical drains: a randomized study. Eur J Cardiothorac Surg 2006;29:562-6.

3. Pawelczyk K, Marciniak M, Kacprzak G, et al. One or two drains after lobectomy? Acomparison of both methods in the immediate postoperative period. Thorac Cardiovasc Surg 2007;55:313-6.

4. Okur E, Baysungur V, Tezel C, et al. Comparison of the single or double chest tube applications after pulmonary lobectomies. Eur J Cardiothorac Surg 2009;35:32-5.

5. Tanaka M, Sagawa M, Usuda K, et al. Postoperative drainage with one chest tube is appropriate for pulmonary lobectomy: a randomized trial. Tohoku J Exp Med 2014;232:55-61.

6. Alex J, Ansari J, Bahalkar P, et al. Comparison of the immediate postoperative outcome of using the conventional two drains versus a single drain after lobectomy. Ann Thorac Surg 2003;76:1046-9.

7. Zhou D, Deng XF, Liu QX, et al. Single chest tube drainage is superior to double chest tube drainage after lobectomy: a meta-analysis. J Cardiothorac Surg 2016;11:88.

8. Zhang X, Lv D, Li M, et al. The single chest tube versus 
double chest tube application after pulmonary lobectomy: A systematic review and meta-analysis. J Cancer Res Ther 2016;12:C309-16.

9. Gonfiotti A, Viggiano D, Voltolini L, et al. Enhanced recovery after surgery and video-assisted thoracic surgery lobectomy: the Italian VATS Group surgical protocol. J Thorac Dis 2018;10:S564-70.

10. Reeb J, Falcoz PE, Olland A, et al. Are daily routine chest radiographs necessary after pulmonary surgery in adult patients? Interact Cardiovasc Thorac Surg 2013;17:995-8.

11. French DG, Dilena M, LaPlante S, et al. Optimizing postoperative care protocols in thoracic surgery: best evidence and new technology. J Thorac Dis 2016;8:S3-11.

12. Drahush N, Miller AD, Smith JS, et al. Standardized Approach to Prolonged Air Leak Reduction After Pulmonary Resection. Ann Thorac Surg 2016;101:2097-101.

13. Younes RN, Gross Jl, Aquiar S, et al. When to remove a CT? A randomized study with subsequent prospective consecutive validation. J Am Coll Surg 2002;195:658-62.

14. Cerfolio RJ, Bryant AS. Results of a prospective algorithm to remove chest tubes after pulmonary resection with high output. J Thorac Cardiovasc Surg 2008;135:269-73.

15. Göttgens KW, Siebenga J, Belgers EH, et al. Early removal of the chest tube after complete video-assisted thoracoscopic lobectomies. Eur J Cardiothorac Surg 2011;39:575-8.

16. Bjerregaard LS, Jensen K, Petersen RH, et al. Early chest tube removal after video-assisted thoracic surgery lobectomy with serous fluid production up to $500 \mathrm{ml} /$ day. Eur J Cardiothorac Surg 2014;45:241-6.

17. Zhang Y, Li H, Hu B, et al. A prospective randomized single-blind control study of volume threshold for chest tube removal following lobectomy. World J Surg 2014;38:60-7.

18. Xie HY, Xu K, Tang JX, et al. A prospective randomized, controlled trial deems a drainage of $300 \mathrm{ml} /$ day safe before removal of the last chest drain after video-assisted thoracoscopic surgery lobectomy. Interact Cardiovasc Thorac Surg 2015;21:200-5.

19. McGuire AL, Petrich W, Maziak DE, et al. Digital versus analogue pleural drainage phase 1: prospective evaluation of interobserver reliability in the assessment of pulmonary air leaks. Interact Cardiovasc Thorac Surg 2015;21:403-7.

20. Cerfolio RJ, Bryant AS. The benefits of continuous and digital air leak assessment after elective pulmonary resection: a prospective study. Ann Thorac Surg 2008;86:396-401.

21. Brunelli A, Salati M, Refai M, et al. Evaluation of a new chest tube removal protocol using digital air leak monitoring after lobectomy: a prospective randomised trial. Eur J Cardiothorac Surg 2010;37:56-60.

22. Filosso PL, Ruffini E, Solidoro P, et al. Digital air leak monitoringafter lobectomy for primary lung cancer in patients with moderate COPD: Does a fast-tracking algorithm reduce postoperative costs and complications? J Cardiovasc Surg 2010;51:1-5.

23. Bertolaccini L, Rizzardi G, Filice MJ, et al. 'Six sigma approach'-an objective strategy in digital assessment of postoperative air leaks: a prospective randomised study. Eur J Cardiothorac Surg 2011;39:e128-32.

24. Pompili C, Detterbeck F, Papagiannopoulos K, et al. Multicenter international randomized comparison of objective and subjective outcomes between electronic and traditional chest drainage systems. Ann Thorac Surg 2014;98:490-6.

25. Filosso PL, Nigra VA, Lanza G, et al. Digital versus traditional air leak evaluation after elective pulmonary resection: a prospective and comparative monoinstitutional study. J Thorac Dis 2015;7:1719-24.

26. Cho HM, Hong YJ, Byun CS, et al. The usefulness of WiFi based digital chest drainage system in the post-operative care of pneumothorax. J Thorac Dis 2016;8:396-402.

27. Lijkendijk M, Licht PB, Neckelmann K. Electronic versus traditional chest tube drainage following lobectomy: a randomized trial. Eur J Cardiothorac Surg 2015;48:893-8.

28. Gilbert S, McGuire AL, Maghera S, et al. Randomized trial of digital versus analog pleural drainage in patients with or without a pulmonary air leak after lung resection. J Thorac Cardiovasc Surg 2015;150:1243-9.

29. Plourde M, Jad A, Dorn P, et al. Digital Air Leak Monitoring for Lung Resection patients: A Randomized Controlled Clinical Trial. Ann Thorac Surg 2018;106:1628-32.

30. Rice TW, Kirby TT. Prolonged air leak. Chest Surg Clin N Am 1992;2:803-11.

31. Marshall MB, Deeb ME, Bleier JI, et al. Suction vs water seal after pulmonary resection: a randomized prospective study. Chest 2002;121:831-5.

32. Cerfolio RJ, Bass C, Katholi CR. Prospective randomized trial compares suction versus water seal for air leaks. Ann Thorac Surg 2001;71:1613-7.

33. Lang P, Manickavasagar M, Burdett C, et al. UK Cardiothoracic Trainees' Research Collaborative. UK Cardiothoracic Trainees\&\#39; Research Collaborative. Suction on chest drains following lung resection: evidence and practice are not aligned. Eur J Cardiothorac Surg 
2016;49:611-6.

34. Alphonso N, Tan C, Utley M, et al. A prospective randomized controlled trial of suction versus non-suction to the under-water seal drains following lung resection. Eur J Cardiothorac Surg 2005;27:391-4.

35. Prokakis C, Koletsis EN, Apostolakis E, et al. Routine suction of intercostal drains is not necessary after lobectomy: a prospective randomized trial. World J Surg 2008;32:2336-42.

36. Brunelli A, Monteverde M, Borri A, et al. Comparison of water seal and suction after pulmonary lobectomy: a prospective, randomized trial. Ann Thorac Surg 2004;77:1932-7.

37. Coughlin SM, Emmerton-Coughlin H, Malthaner R. Management of chest tubes after pulmonary resection: a systematic review and meta-analysis. Can J Surg 2012;55:264-70.

38. Deng B, Tan QY, Zhao YP, et al. Suction or non-suction to the underwater seal drains following pulmonary operation: meta-analysis of randomized controlled trials. Eur J Cardiothorac Surg 2010;38:210-5.

39. Liberman M, Muzikansky A, Wright CD, et al. Incidence and risk factors of persistent air leak after major pulmonary resection and use of chemical pleurodesis. Ann Thorac Surg 2010;89:891-7.

40. Manley K, Coonar A, Wells F, et al. Blood patch for persistent air leak: a review of the current literature. Curr Opin Pulm Med 2012;18:333-8.

Cite this article as: French DG, Gilbert S. Technology and evidence-based care enhance postoperative management of chest drains. J Thorac Dis 2018;10(12):6399-6403. doi: 10.21037/jtd.2018.11.99
41. Andreetti C, Venuta F, Anile M, et al. Pleurodesis with an autologous blood patch to prevent persistent air leaks after lobectomy. J Thorac Cardiovasc Surg 2007;133:759-62.

42. Chambers A, Routledge T, Billè A, et al. Is blood pleurodesis effective for determining the cessation of persistent air leak? Interact Cardiovasc Thorac Surg 2010;11:468-72.

43. Dugan KC, Laxmanan B, Murgu S, et al. Management of Persistent Air Leaks. Chest 2017;152:417-23.

44. Mahajan AK, Khandhar SJ. Bronchoscopic valves for prolonged air leak: current status and technique. J Thorac Dis 2017;9:S110-5.

45. Merritt RE, Singhal S, Shrager JB. Evidence-based suggestions for management of air leaks. Thorac Surg Clin 2010;20:435-48.

46. Rieger KM, Wroblewski HA, Brooks JA, et al. Postoperative outpatient chest tube management: initial experience with a new portable system. Ann Thorac Surg 2007;84:630-2.

47. Royer AM, Smith JS, Miller A, et al. Safety of outpatient chest tube management of air leaks after pulmonary resection. Am Surg 2015;81:760-3.

48. Schmocker RK, Vanness DJ, Macke RA, et al. Outpatient air leak management after lobectomy: a CMS cost analysis. The Journal of surgical research 2016;203:390-7.

49. Varela G, Jimenez MF, Novoa N. Portable chest drainage systems and outpatient chest tube management. Thorac Surg Clin 2010;20:421-6. 